\title{
Axons Giving Rise to the Palisade Endings of Feline Extraocular Muscles Display Motor Features
}

\author{
Lars Zimmermann, ${ }^{1}$ Camilo J. Morado-Díaz, ${ }^{2}$ María A. Davis-López de Carrizosa, ${ }^{2}$ Rosa R. de la Cruz, ${ }^{2}$ Paul J. May, ${ }^{3}$ \\ Johannes Streicher, ${ }^{1}$ Ángel M. Pastor, ${ }^{2 *}$ and Roland Blumer ${ }^{1 *}$ \\ ${ }^{1}$ Center of Anatomy and Cell Biology, Integrative Morphology Group, Medical University Vienna, A-1090 Vienna, Austria, ${ }^{2}$ Department of Physiology, \\ Faculty of Biology, University of Seville, 41012 Seville, Spain, and ${ }^{3}$ Department of Neurobiology and Anatomical Sciences, University of Mississippi Medical \\ Center, Jackson, Mississippi 39216
}

Palisade endings are nerve specializations found in the extraocular muscles (EOMs) of mammals, including primates. They have long been postulated to be proprioceptors. It was recently demonstrated that palisade endings are cholinergic and that in monkeys they originate from the EOM motor nuclei. Nevertheless, there is considerable difference of opinion concerning the nature of palisade ending function.

Palisade endings in EOMs were examined in cats to test whether they display motor or sensory characteristics. We injected an anterograde tracer into the oculomotor or abducens nuclei and combined tracer visualization with immunohistochemistry and $\alpha$-bungarotoxin staining. Employing immunohistochemistry, we performed molecular analyses of palisade endings and trigeminal ganglia to determine whether cat palisade endings are a cholinergic trigeminal projection.

We confirmed that palisade endings are cholinergic and showed, for the first time, that they, like extraocular motoneurons, are also immunoreactive for calcitonin gene-related peptide. Following tracer injection into the EOM nuclei, we observed tracer-positive palisade endings that exhibited choline acetyl transferase immunoreactivity. The tracer-positive nerve fibers supplying palisade endings also established motor terminals along the muscle fibers, as demonstrated by $\alpha$-bungarotoxin. Neither the trigeminal ganglion nor the ophthalmic branch of the trigeminal nerve contained cholinergic elements.

This study confirms that palisade endings originate in the EOM motor nuclei and further indicates that they are extensions of the axons supplying the muscle fiber related to the palisade. The present work excludes the possibility that they receive cholinergic trigeminal projections. These findings call into doubt the proposed proprioceptive function of palisade endings.

\section{Introduction}

To localize the position of objects in the surrounding visual space, the CNS needs visual information from the retina and additional information about eye position in the orbit. It is believed that such nonvisual eye position information comes from sense organs (proprioceptors) within the extraocular muscles (EOMs). However, in the EOMs of mammals, classical proprioceptors (muscle spindles and Golgi tendon organs) are absent in most species except even-toed ungulates (Maier et al., 1974). The reason for these interspecies variations is unclear. Another structure, unique to EOMs, has been found in all mammalian species thus

\footnotetext{
Received Aug. 28, 2012; revised Dec. 6, 2012; accepted Dec. 11, 2012.

Author contributions: R.B., A.M.P., R.R.C., and P.J.M. designed research; L.Z., C.J.M., M.A.D., A.M.P., and R.B. performed research; L.Z., R.R.C., A.M.P., and R.B. analyzed data; L.Z., R.R.C., P.J.M., J.S., A.M.P., and R.B. wrote the paper.

This study was supported by Grant P20881-B09 from Fonds zur Foerderung der Wissenschaftlichen Forschung, Austria-Spain concerted actions ESO4/2010 and AT2009-0039 to A.M.P. and R.B., and grants BFU2009-07121 and BFU2012-33975 from MEC-FEDER and CVI-6053 from Junta de Andalucía-FEDER to A.M.P. We thank Regina Mayer, Marietta Lipowec, and Marlene Rodler for their valuable technical assistance.

${ }^{*}$ A.M.P. and R.B. are co-senior authors.

Correspondence should be addressed to Dr. Roland Blumer, Center of Anatomy and Cell Biology, Integrative Morphology Group, Medical University Vienna, Waehringer Strasse 13, 1090 Vienna, Austria. E-mail: roland.blumer@meduniwien.ac.at.

DOI:10.1523/JNEUROSCI.4116-12.2013

Copyright (C) 2013 the authors $\quad 0270-6474 / 13 / 332784-10 \$ 15.00 / 0$
}

far investigated - the palisade ending, also called the innervated myotendinous cylinder (Dogiel, 1906; Ruskell, 1978; AlvaradoMallart and Pinçon-Raymond, 1979). Palisade endings are encapsulated nervous end organs and arise from axons coming from the muscle belly and entering the proximal and distal tendons. The axons turn back $180^{\circ}$ within the tendon to terminate in a cuff of nerve terminals around the tip of a single muscle fiber. The muscle fibers associated with palisade endings are the multiply innervated muscle fibers (MIFs) of the global layer of EOMs. These possess several en grappe nerve endings along their length (Ruskell, 1978; Alvarado-Mallart and Pinçon-Raymond, 1979).

The functional significance of palisade endings is still a matter of discussion. It is not clear whether they are sensory (Ruskell, 1978; Alvarado-Mallart and Pinçon-Raymond, 1979; Billig et al., 1997; Büttner-Ennever et al., 2003), motor (Sas and Scháb, 1952; Konakci et al., 2005a; Blumer et al., 2009), or both (Lukas et al., 2000). Arguments for a sensory role arise from their peculiar location at the muscle-tendon transition (Ruskell, 1978; Alvarado-Mallart and Pinçon-Raymond, 1979), their similarities to Golgi tendon organs (Zelená and Soukup, 1977), and a report in cats of anterograde labeling from the sensory trigeminal ganglion (Billig et al., 1997). Evidence that palisade endings are motor is also available in the literature. Palisade endings degenerate after lesions of the oculomotor nuclei and have a cholinergic 
Table 1. List of antibodies and other markers, including their working dilution, used in this study

\begin{tabular}{lll}
\hline Primary antibodies (Millipore) & Secondary antibodies (Invitrogen) & 0ther markers $\left(\right.$ Sigma $^{1}{ }^{1}$ Invitrogen $\left.^{2}\right)$ \\
\hline Goat anti-choline acetyltransferase (1:100) & Rabbit anti-goat Alexa Fluor 488 (1:500/1:300) & Phalloidin Atto647 (1:100) ${ }^{1}$ \\
Chicken anti-neurofilament (1:500/1:300) & Donkey anti-goat Alexa Fluor 568 (1:500/1:300) & $\alpha$-bungarotoxin rhodamine/Alexa Fluor 488 (1:500/1:300) ${ }^{1}$ \\
Rabbit anti-CGRP (1:400/1:300) & Goat anti-chicken Alexa Fluor 568 (1:500/1:300) & Avidin Alexa Fluor 488 $(1: 250 / 1: 150)^{2}$ \\
Mouse anti-synaptophysin (1:500/1:200) & Goat anti-rabbit Alexa Fluor 488 (1:500/1:300) & Streptavidin Alexa Fluor 568 $(1: 500 / 1: 300)^{2}$ \\
& Goat anti-mouse Alexa Fluor 488 (1:500/1:300) & Streptavidin-HRP $(1: 1000 / 1: 600)^{2}$ \\
\hline
\end{tabular}

The first working dilution within the bracket is for the staining of sections of the muscle belly, the second working dilution is for staining of the whole mounts.
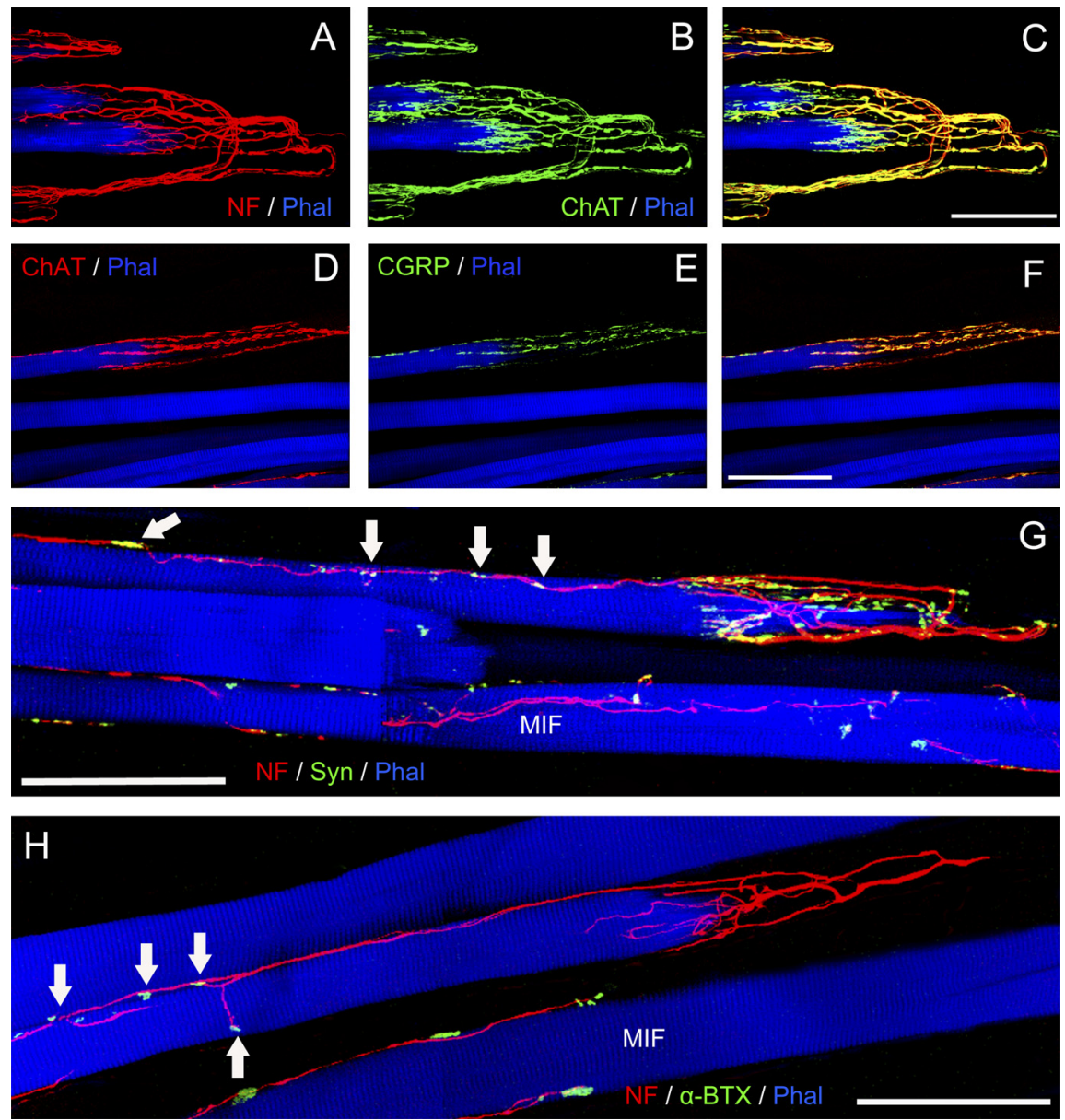

Figure 1. Three-dimensional projections of stacks of CLSM images showing triple fluorescence staining of palisade endings in the muscle-tendon transition of whole-mount preparations. $\boldsymbol{A}-\boldsymbol{H}$, All palisade endings exhibit a characteristic shape; nerve fibers extending along the muscle fibers reach onto the tendon, where they turn back $180^{\circ}$ to terminate in arborizations at the tip of a single muscle fiber. The tendon is not visualized and continues to the right of the muscle fibers. $\boldsymbol{A}-\boldsymbol{C}$, Palisade endings are supplied by a bundle of axons. $\boldsymbol{D}-\boldsymbol{H}$, Palisade endings are supplied by a single axon. $\boldsymbol{A}$-C, Staining of a medial rectus muscle with anti-neurofilament (red), anti-ChAT (green), and phalloidin (blue). $\boldsymbol{A}, \boldsymbol{B}$, Palisade endings showing neurofilament immunoreactivity $(\boldsymbol{A})$ and ChAT immunoreactivity $(\boldsymbol{B})$. $\boldsymbol{C}, 0$ verlay of $\boldsymbol{A}$ and $\boldsymbol{B}$ appears in a yellow mixed color showing that all palisade endings exhibit neurofilament/ChAT immunoreactivity. $\boldsymbol{D}-\boldsymbol{F}$, Staining of a superior rectus muscle with anti-ChAT (red), anti-CGRP (green), and phalloidin (blue). D, E, A palisade ending exhibiting ChAT immunoreactivity $(\boldsymbol{D})$ and $C G R P$ immunoreactivity $(\boldsymbol{E})$. $\boldsymbol{F}$, Overlay of $\boldsymbol{D}$ and $\boldsymbol{E}$ showing the palisade in a yellow mixed color. $\boldsymbol{G}$, Staining of a medial rectus muscle with anti-neurofilament (red), anti-synaptophysin (green), and phalloidin (blue). A nerve fiber forming a palisade ending establishes neuromuscular contacts (arrows) along the muscle fiber. Palisade nerve terminals and neuromuscular contacts are synaptophysin immunoreactive. Neuromuscular contacts outside the palisade complex resemble en grappe motor terminals of the multiply innervated muscle fiber, MIF. $\boldsymbol{H}$, Staining of an oblique inferior muscle with anti-neurofilament (red), $\alpha$-bungarotoxin (green), and phalloidin (blue). The nerve fiber supplying the palisade ending establishes $\alpha$-bungarotoxin-positive motor contacts (arrows) along the muscle fiber. Note that unlike other terminals the axons makes on the fiber, palisade nerve terminals lack $\alpha$-bungarotoxin staining. In addition to the MIF with a palisade ending, a second MIF is labeled in $\boldsymbol{G}$ and $\boldsymbol{H}$. Scale bars, $100 \mu \mathrm{m}$.

phenotype. Furthermore, in 30\% of the cases, axons supplying monkey palisade endings establish additional motor terminals along the muscle fibers (Sas and Scháb, 1952; Konakci et al., 2005a; Konakci et al., 2005b; Blumer et al., 2009). Recently, palisade endings in monkey have been anterogradely labeled after tracer injection into the EOM motor nuclei (Lienbacher et al., 2011a; Zimmermann et al., 2011). In a subsequent report, Lienbacher et al. (2011b) suggested that despite this origin, palisade endings are sensory and arise from sensory-like neurons detected around the periphery of the oculomotor nucleus.

Here, we analyzed palisade endings in cats to test whether these structures exhibit motor as opposed to sensory features. First, we examined the molecular characteristics of palisade endings by use of calcitonin gene-related peptide (CGRP) detection. Second, we made central tracer injections into EOM motor nuclei and combined the anterograde labeling of axons with choline acetyl transferase immunohistochemistry and $\alpha$-bungarotoxin staining. Third, we examined whether palisade endings receive cholinergic trigeminal projections.

\section{Materials and Methods}

In the present study, four adult female cats weighing between 2.0 and $2.5 \mathrm{~kg}$ were used for central tracer injections. Surgical and handling procedures for experiments followed the guidelines of the National Institute of Health (http:/oacu.od.nih.gov) and specific recommendations for maintenance of higher mammals during neuroscience experiments $(\mathrm{NIH}$ publication no. 94-3207,1994) and were in accordance with national legislation for the use and care of laboratory animals (R.D. 120/2005 BOE 252/34367-91,2005).

\section{Tracer injection}

In two cats (cat 1 and 2) the anterograde tracer, biotinylated dextran amine (BDA), was injected bilaterally into the oculomotor nuclei, and in two other cats (cat 3 and 4) BDA was injected bilaterally into the abducens nuclei.

\section{Surgical procedure}

Cats were anesthetized (sodium pentobarbital, $35 \mathrm{mg} / \mathrm{kg}$, i.p.) and prepared for BDA injection. Bipolar electrodes were implanted stereotaxically in the abducens or the oculomotor nerve at its exit from the brainstem to identify the corresponding motor nucleus by locating the antidromically evoked field potential before injection. Electrical stimulation consisted of square wave single pulses of $50 \mu$ s duration and $<50 \mu \mathrm{A}$ intensity. BDA was electrophoretically injected into either the abducens or the oculomotor nuclei at sites 
where evoked potentials were recorded. Glass micropipettes bevelled to a tip diameter of 15 $\mu \mathrm{m}$ were filled with a solution of $10 \%$ BDA in 0.05 м Tris- $\mathrm{HCl}$ buffer ( $\mathrm{pH}$ 7.4). Positive current pulses of $7 \mu \mathrm{A}$ intensity were delivered at $50 \%$ duty cycle (7 s on, $7 \mathrm{~s}$ off) for $20 \mathrm{~min}$ at three different rostrocaudal locations. Animals were monitored daily after surgery and postoperative care was provided using analgesics ( $\mathrm{pi}$ razolone, $0.1 \mathrm{~g} / \mathrm{kg} / \mathrm{d}$, i.m.) and antibiotics (streptomycin and penicillin, 20,000 IU/kg/d, i.m.) during the initial 3 days.

Following a survival time of 3 weeks, the animals were deeply anesthetized with a terminal dose of sodium pentobarbital. The ascending aorta was cannulated and the circulatory system was rinsed with Ringer solution, followed by perfusion fixation with $4 \%$ paraformaldehyde in $0.1 \mathrm{~m}$ sodium phosphate buffer, $\mathrm{pH}$ 7.4. The brainstem, the trigeminal ganglia, including the ophthalmic, maxillary, and mandibular nerves, and the EOMs were removed. The tissue was immersion fixed for $2 \mathrm{~h}$ in the above fixative and then stored in buffer $\left(4^{\circ} \mathrm{C}\right)$ before processing.

The EOMs were cut transversally into two pieces: one piece containing the muscle belly and the second containing the distal tendon, including the muscle-tendon transition. The distal myotendons of the EOMs were cryoprotected and frozen as whole-mount preparations. The muscle bellies and the trigeminal ganglia were also cryoprotected, frozen, and cut $10 \mu \mathrm{m}$ thick with a microtome (CM1950, Leica). The muscle bellies were cut lengthwise, parallel to the orbital surface of the muscle, and the trigeminal ganglia were cut roughly in the frontal plane. Sections were mounted onto glass slides (SuperFrost Ultra Plus, Fisher Scientific). Brainstem coronal sections were cut at $50 \mu \mathrm{m}$ with a vibratome (VT 1000S, Leica) and further processed, free-floating, for the detection of the injection site.

EOM whole-mounts and tissue sections were processed for fluorescence labeling, tracer detection, or alternatively for combinations of tracer detection and fluorescence labeling. In particular, the nontracer labeled EOMs of the oculomotor/abducens cases and the trigeminal ganglia were used for fluorescence labeling. The brainstems and tracer labeled EOMs (muscle bellies and musculotendinous transitions) were used for single tracer detection or alternatively for tracer detection combined with fluorescence labeling.

\section{Immunofluorescence labeling}

Sections of the trigeminal ganglion were labeled with antibodies against choline acetyltransferase (ChAT) and neurofilament proteins or antibodies against CGRP and neurofilaments. EOM whole-mounts (distal EOM myotendons) from muscles that did not contain BDA-positive axons were labeled with four combinations of triple-fluorescent labeling: (1) antibodies against ChAT and neurofilaments and with phalloidin (used as counterstaining of the muscle fibers); (2) antibodies against ChAT and CGRP and with phalloidin; (3) antibodies against neurofilaments and synaptophysin and with phalloidin; and (4) antibody against neurofilaments and $\alpha$-bungarotoxin and with phalloidin. The sources and working dilutions of phalloidin, $\alpha$-bungarotoxin, primary antibodies, and secondary antibodies are listed in Table 1.

Tissue was blocked for $2 \mathrm{~h}$ with $10 \%$ serum (according to the secondary antibodies) in sodium PBS containing Triton X-100 (PBS-T): 0.1\%
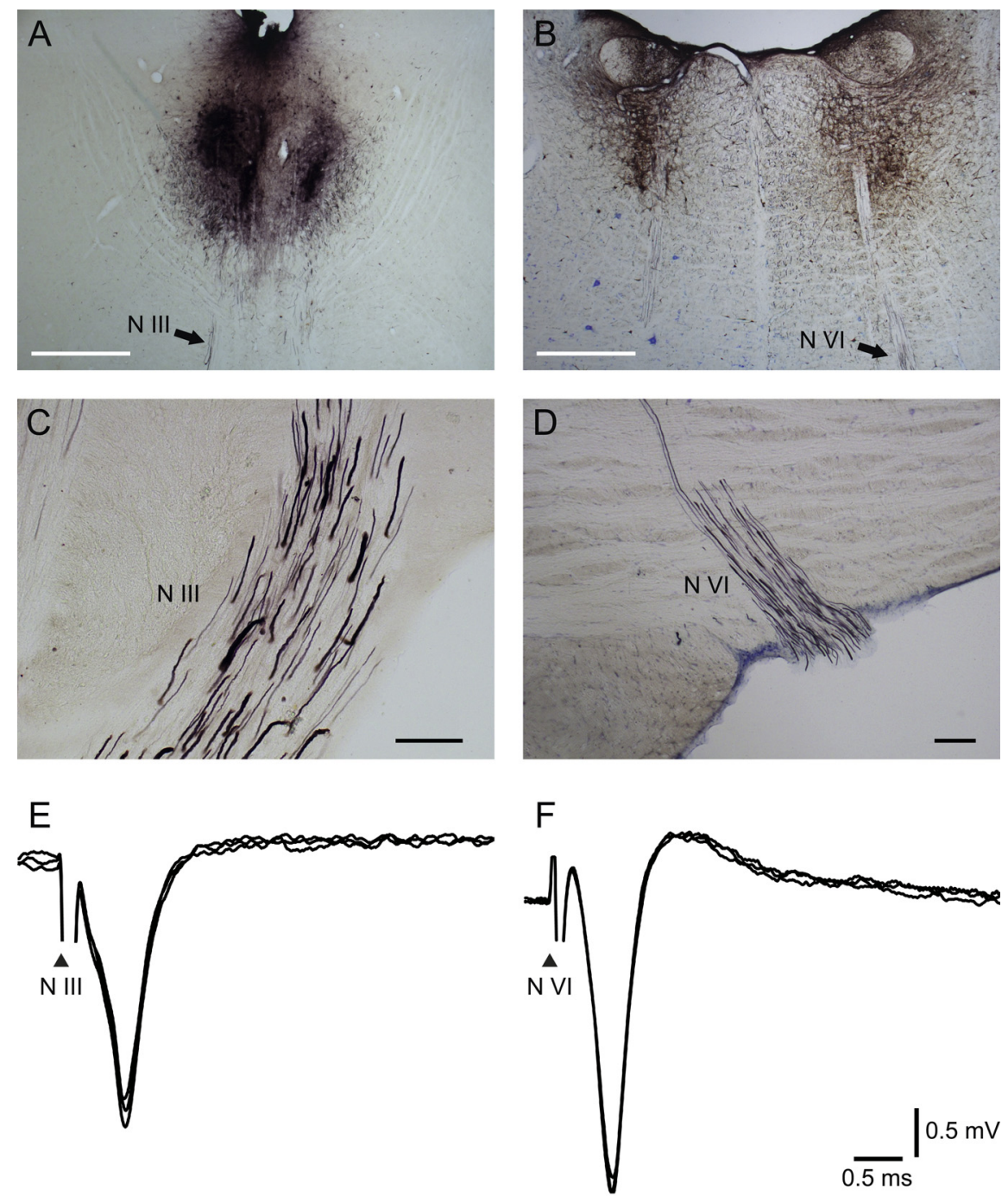

Figure 2. BDA tracer injections and labeling in the brainstem. The tracer was visualized with the DAB reaction and enhanced with ammonium sulfate, and so it appears in black. $\boldsymbol{A}, \boldsymbol{B}$, Image of the injection site in the oculomotor nucleus $(\boldsymbol{A})$ and in the scale bars, $1 \mathrm{~mm}$. C, D, Rootlets of the oculomotor nerve (N III) ( $(\boldsymbol{C})$ and the abducens nerve (N VI) (D) show imulation applied to the IIIrd $(\boldsymbol{E})$ and VIth $(\boldsymbol{F})$ nerves, respectively. Arrowhead denotes the stimulus artifact produced by the electrical stimulation to the corresponding nerve.

Triton X-100 for trigeminal ganglion sections or $1 \%$ Triton X-100 for EOM whole-mounts. Then, the tissue was incubated for $48 \mathrm{~h}$ with the primary antibodies, rinsed thoroughly with PBS-T, and incubated with one of the secondary antibodies for $2 \mathrm{~h}$ for sections and $24 \mathrm{~h}$ for whole mounts. After another washing step, tissue was incubated with the other secondary antibody and phalloidin overnight (staining combinations 1-3) or with $\alpha$-bungarotoxin and phalloidin (staining combination 4 ). Finally, the tissue was rinsed again and mounted in $60 \%$ glycerine (v/v) plus $40 \%$ PBS.

For negative control experiments, primary antibodies were omitted and secondary antibodies were used alone. In all cases, the omission of the primary antibodies resulted in a complete lack of immunostaining.

\section{Single-color and multicolor tracer detection}

In single-color preparations the tracer was visualized using conventional avidin-biotin procedures. Alternatively, in multicolor preparations tracer visualization with fluorescent avidin/streptavidin was combined with additional fluorescent staining of cellular markers.

Single-color detection. Sections of the brainstem and EOM muscle bellies, as well as muscle/tendon whole mounts, were blocked in $5 \% \mathrm{H}_{2} \mathrm{O}_{2}$ and incubated with horseradish peroxidase-conjugated streptavidin 
Table 2. Percentage of tracer-positive axons in the extraocular muscles of cats 1, 2, 3 , and 4

\begin{tabular}{|c|c|c|c|c|}
\hline \multirow[b]{2}{*}{ EOM } & \multicolumn{2}{|c|}{ Oculomotor injections } & \multicolumn{2}{|c|}{ Abducens injections } \\
\hline & Cat $1^{a}$ & ${\operatorname{Cat~} 2^{a}}^{a}$ & Cat $3^{a}$ & Cat $4^{a}$ \\
\hline SR le/rii & 22/16 & 17/15 & $-1-$ & $-1-$ \\
\hline IR le/rib & $55 / 58$ & 49/73 & $-1-$ & $-1-$ \\
\hline LR le/rib & $-1-$ & $-1-$ & $64 / 46$ & $51 / 31$ \\
\hline MR le/ri ${ }^{b}$ & $47 / 53$ & $47 / 36$ & $-1-$ & $-1-$ \\
\hline SOle/ri ${ }^{b}$ & $-1-$ & $-1-$ & $-1-$ & $-1-$ \\
\hline $10 \mathrm{le} / \mathrm{ri}^{b}$ & $14 / 28$ & $39 / 12$ & $-1-$ & $-1-$ \\
\hline LPS le/rib & $10 / 6$ & $5 / 6$ & $-1-$ & $-1-$ \\
\hline
\end{tabular}

a Percentage of tracer-positive axons.

${ }^{b}$ Abbreviations indicate the following muscles: $S R$, Superior rectus; IR, inferior rectus; $L R$, lateral rectus; $M R$, medial rectus: $\mathrm{SO}$, superior oblique; 10 , inferior oblique, LPS, levator palpebrae superioris. Left and right are indicated as le and ri, respectively.
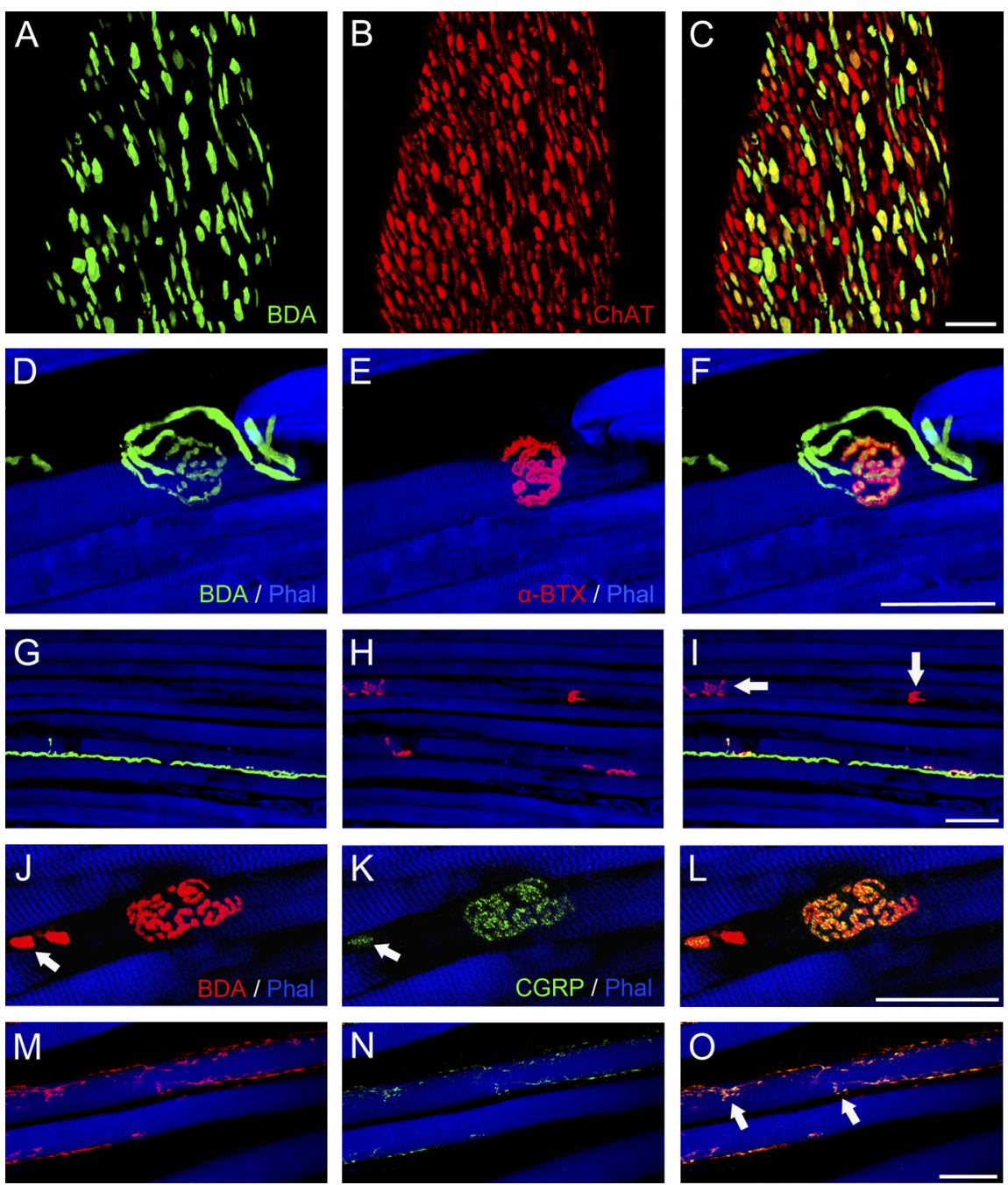

Figure 3. Three-dimensional projections from stacks of CLSM images showing BDA tracer combined with fluorescence staining of other cellular molecules. $\boldsymbol{A}-\boldsymbol{C}$, Tracer visualization with avidin (green) was combined with anti-ChAT labeling (red). $\boldsymbol{A}$, Tracerpositive axons at the entry site of the abducens nerve in the lateral rectus muscle. $\boldsymbol{B}$, The axons of this abducens nerve exhibit ChAT immunoreactivity. $\boldsymbol{C}$, Overlay of $\boldsymbol{A}$ and $\boldsymbol{B}$ in yellow mixed color showing that all tracer-positive axons also exhibit ChAT immunoreactivity, but other ChAT-positive axons contained no tracer. $\boldsymbol{D}-\boldsymbol{I}$, Triple fluorescence staining with streptavidin (green), $\alpha$-bungarotoxin (red), and phalloidin (blue) of a medial rectus muscle. $\boldsymbol{D}, \mathbf{G}$, Tracer-positive axons establishing neuromuscular contacts resembling en plaque motor terminals $(\boldsymbol{D})$ and en grappe motor terminals $(\boldsymbol{G}) . \boldsymbol{E}, \boldsymbol{H}$, En plaque and en grappe motor terminals, respectively, after $\alpha$-bungarotoxin labeling. $F, I$, Overlays in yellow mixed color confirming that tracer-positive en plaque-like and en grape-like endings bind $\alpha$-bungarotoxin. Other motor terminals (arrows) lack tracer $(\boldsymbol{I})$. $\boldsymbol{J}-\mathbf{0}$, Triple fluorescence staining with streptavidin (red), anti-CGRP (green), and phalloidin (blue) $J, M$, Tracer-positive axons establishing en plaque and en grappe motor terminals, respectively. $\boldsymbol{K}, \boldsymbol{N}$, Tracer-positive axons and motor terminals exhibit CGRP immunoreactivity. The arrows in $\boldsymbol{J}$ and $\boldsymbol{K}$ show the tracer (BDA)-positive axon (in $\boldsymbol{J}$ ), which also showed (GRP immunolabeling (in $\boldsymbol{K}$ ). $\boldsymbol{L}, \mathbf{0}, 0$ verlays in yellow mixed color. En grappe endings are indicated by arrows $(\mathbf{0})$. Scale bars, $50 \mu \mathrm{m}$.
(Dako) ( $2 \mathrm{~h}$ for sections and $48 \mathrm{~h}$ for whole mounts). Following several washes in PBS, tissue was incubated in a freshly prepared solution containing 3.3'-diaminobenzidine tetrahydrochloride and $0.06 \% \mathrm{H}_{2} \mathrm{O}_{2}$. In medium to obtain an intensified black reaction product.

Multicolor detection. Sections of the EOM muscle bellies and EOM In staining combinations 1,2 and 3 , tissue was blocked in $10 \%$ serum (according to the secondary antibodies) and then incubated for $48 \mathrm{~h}$ with the primary antibodies. Following rinsing in PBS-T, tissue was incubated with the corresponding secondary antibodies for $2 \mathrm{~h}$ (sections) or $24 \mathrm{~h}$ (whole-mounts). Then, tissue was incubated for $2 \mathrm{~h}$ (sections) or $24 \mathrm{~h}$ (whole-mounts) with streptavidin/avidin and phalloidin. In staining combination 4, tissue was incubated in a solution containing $\alpha$-bungarotoxin (conjugated with Alexa Fluor 488), streptavidin (with Alexa Fluor 568), and phalloidin (with Alexa Fluor 633) for $2 \mathrm{~h}$ (sections) or $24 \mathrm{~h}$ (whole-mounts).

\section{Data analysis}

DAB-labeled sections and whole mounts were analyzed with a light microscope (Zeiss Axioscope) and images were taken with a Nikon Eclipse 600 camera. Images of palisade endings were recorded at different focal depths $(1 \mu \mathrm{m})$, and $3 \mathrm{D}$ projections were obtained using the software Lucia G (Nikon, Düsseldorf).

Fluorescently labeled sections and whole mounts were analyzed with a confocal laser scanning microscope (CLSM; LSM 510, Carl Zeiss Meditec). Series of virtual CLSM sections of $0.5-1 \mu \mathrm{m}$ thickness were cut through the structures of interest. Each section was photo-documented and $3 \mathrm{D}$ projections were formulated on a computer using LSM Image Examiner software (Carl Zeiss Meditec). Images were generated in three different fluorescence channels with excitation wavelengths of 488,568 , and $633 \mathrm{~nm}$. In some cases a transmission light image was recorded instead of the image with $633 \mathrm{~nm}$ excitation.

\section{Results}

Morphology of palisade endings

Palisade endings were analyzed in EOM whole mounts of the distal muscle tendon transition. Such whole-mount preparations allow study of the overall morphology of palisade endings. We observed palisade endings in each EOM. All palisade endings exhibited a typical structure; axons originating from the muscle belly extended onto the tendon, where they turned back $180^{\circ}$ to terminate in a cuff of terminals around the tip of a single muscle fiber. Usually, palisade 
endings were formed by single axons (Fig. $1 D-H)$, but occasionally we also found palisade endings that were supplied by two or more nerve fibers (Fig. $1 A-C$ ).

When possible, nerve fibers supplying palisade endings were traced back from their terminal branches in the tendon toward the muscle. We observed axons supplying palisade endings that established contacts along the muscle fibers (Fig. $1 G, H)$. Such additional neuromuscular contacts resembled en grappe motor endings of MIF and were observed in $25 \%$ of the palisade endings. In the remaining cases it was not possible to evaluate whether axons supplying palisades established additional neuromuscular contacts, either because the axon-forming palisades intermingled with other axons or because it was not running alongside the muscle fiber and approached the palisade laterally (Fig. $1 A-C$ ).

\section{Palisade endings exhibit ChAT/CGRP} immunoreactivity and are supplied by axons that establish $\alpha$-bungarotoxin-positive contacts along the muscle fibers To determine the molecular characteristics of palisade endings, muscle/tendon whole mounts were triple fluorescent-stained with different combination of markers for cellular molecules. We used antibodies against ChAT, CGRP, synaptophysin, and neurofilament as well as $\alpha$-bungarotoxin labeling. Phalloidin was used to stain muscle fibers.

It was demonstrated that axons supplying palisade endings exhibited ChAT/neurofilament immunoreactivity. In those cases in which palisade endings were supplied by multiple axons, all axons were ChAT/neurofilament positive (Fig. $1 A-C$ ). Moreover, all palisade endings exhibited CGRP immunoreactivity (Fig. $1 D-F$ ), analogous to that observed in motor axons and en plaque and en grappe motor endings (see below, next section, fourth paragraph). Palisade nerve terminals were positive for synaptophysin (a marker for all kinds of nerve synaptic terminals; Fig. $1 G$ ) but lacked $\alpha$-bungarotoxin staining (the classical marker for motor nerve terminals; Fig. $1 H$ ). Since $\alpha$-bungarotoxin labels nicotinic acetylcholine receptors on the muscle fiber membrane, this finding suggests that palisade nerve terminals established contacts on the tendon and not on the muscle fibers. This is in line with ultrastructural studies in which most palisade nerve terminals were observed to contact the tendon, and palisade nerve terminals contacting the muscle fiber were an exceptional feature ( Alvarado-Mallart and Pinçon-Raymond, 1979; Konakci et al., 2005a). On the other hand, additional neuromuscular contacts along the muscle fibers made by the same axon that supplied the palisade endings were positive for synaptophysin and $\alpha$-bungarotoxin as well (Fig. $1 G, 1 \mathrm{H}$ ).

\section{Tracer injection into the oculomotor and abducens nucleus labels en grappe motor terminals and palisade endings arising from the same parent axons}

To examine whether palisade endings originate from EOM motor nuclei, we injected the anterograde tracer BDA into either the oculomotor or the abducens nucleus. These nuclei were located with the aid of the antidromic field potential elicited by electrical stimulation applied to the IIIth and VIth nerves, respectively (Fig. 2E,F).
Following the oculomotor injections, tracer uptake involved all subgroups of the oculomotor nucleus (Fig. 2A). In the abducens cases, tracer uptake involved the entire abducens nucleus (Fig. 2B). Many tracer-positive axons were observed in the rootlets of the oculomotor and abducens nerves (Fig. 2C,D).

In the muscle bellies, tracer-labeled axons and nerve terminals were observed in the corresponding EOMs. The levator palpebrae superioris, which is innervated by the oculomotor nerve, likewise contained tracer-positive axons. The number of tracerpositive axons varied in the EOMs. In both oculomotor cases, the inferior rectus and the medial rectus on both sides contained a higher number of tracer-positive axons (between 36 and 73\%) than the superior rectus and the inferior oblique (between 15 and $28 \%)$. An exception was the left inferior oblique of cat 2 in which $39 \%$ of the axons contained tracer. In the abducens cases, $51-$ $64 \%$ of the axons were labeled in the left lateral rectus, and 31$46 \%$ in the right lateral rectus muscle. The specific percentage of tracer-labeled axons per EOM in each case is given in Table 2.

Combinations of BDA/anti-ChAT or alternatively $\mathrm{BDA} / \alpha$ bungarotoxin labeling showed that all tracer-positive axons entering the EOMs exhibited ChAT immunoreactivity (Fig. 3A-C). These BDA- labeled axons established $\alpha$-bungarotoxin-positive motor contacts via en plaque endings on singly innervated muscle fibers (SIFs) (Fig. 3D-F) and via en grappe endings on MIFs (Figs. $3 G-I$ ). We also detected ChAT-positive axons (Fig. 3C), $\alpha$-bungarotoxin-positive en plaque terminals (data not shown), and $\alpha$-bungarotoxin-positive en grappe motor terminals (Fig. 3C, arrows) that lacked tracers. Combination of BDA/anti-CGRP showed that tracer-positive axons and en plaque and en grappe motor endings were CGRP positive (Fig. 3J-O), like those seen in the palisade endings (Fig. $1 D-F$ ).

Next, we examined muscle tendon transitions of EOMs to determine whether palisade endings were anterogradely labeled from the oculomotor or abducens nucleus. In single color labeling, the tracer was visualized with a DAB reaction (Fig. 4), and in multicolor labeling, tracer visualization was combined with immunolabeling (anti-synaptophysin, antiChAT) and $\alpha$-bungarotoxin labeling (Fig. 5). Muscle fibers were stained with phalloidin. 

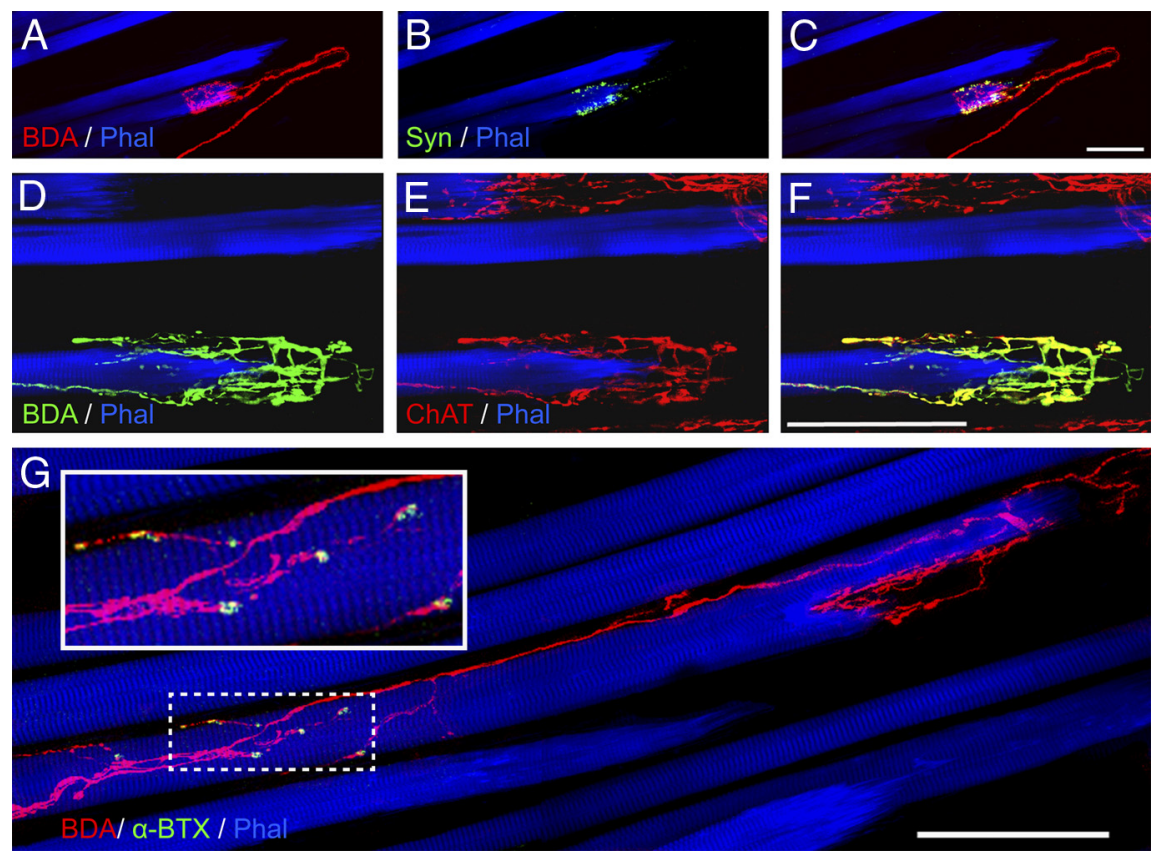

Figure 5. Three-dimensional projections of stacks of CLSM images showing tracer-labeled palisade endings in muscle-tendon, whole-mount preparations. Tracer visualization was combined with fluorescence staining of other cellular molecules. $\boldsymbol{A}-\boldsymbol{C}$, Palisade ending in a lateral rectus muscle. The visualization of the anterograde tracer BDA with streptavidin (red) was combined anti-synaptophysin (green) and phalloidin (blue). $\boldsymbol{A}$, A tracer-positive palisade ending. $\boldsymbol{B}$, Palisade nerve terminals exhibit synaptophysin immunoreactivity. $\boldsymbol{C}$, Overlay of $\boldsymbol{A}$ and $\boldsymbol{B}$. Palisade nerve terminals appear in yellow mixed color. $\boldsymbol{D}-\boldsymbol{F}$, Palisade endings in a superior rectus muscle. Tracer visualization with avidin (green) was combined with anti-ChAT (red) and phalloidin (blue). D, A single tracer-positive palisade ending. $\boldsymbol{E}$, Demonstrating two ChAT-positive palisade endings. $\boldsymbol{F}$, Overlay in yellow mixed color exhibiting that tracer-positive palisade ending is ChAT-positive as well. The ChAT-positive palisade ending in the upper part of the image lacks tracer. G, Palisade ending in an inferior rectus muscle. Tracer visualization with streptavidin (red) was combined with $\alpha$-bungarotoxin (green) and phalloidin (blue). The tracer-positive palisade ending is supplied by a nerve fiber that forms $\alpha$-bungarotoxin-positive motor contacts along the muscle fiber. A detail of the motor contacts is shown in the inset. Scale bars, $100 \mu \mathrm{m}$.

We found tracer-positive palisade endings exhibiting their typical morphology after both oculomotor and abducens BDA injections (Figs. 4, 5). All tracer-positive palisade endings exhibited synaptophysin immunoreactivity (Fig. $5 \mathrm{~A}-\mathrm{C}$ ) and ChAT immunoreactivity as well (Fig. $5 D-F$ ). We also observed synaptophysin/ ChAT-immunoreactive palisades that contained no tracer (Fig. $5, E, F$, top). An additional striking finding was that the tracerpositive axons that formed palisades also established en grappe nerve terminals along the muscle fibers (Fig. $4 C$ and zfr;5Fig. 5G). These resembled the pattern of connections found in MIFs (Fig. 1G,H). The neuromuscular contacts bound $\alpha$-bungarotoxin, although the palisade terminals did not (Fig. $5 G$ ).

In total, we counted 120 tracer-positive palisade endings, the number varying between 1 and 13 per EOM. However, labeling was never found in muscles not supplied by the injected nucleus. The quantity of tracer-positive palisades for each case and each corresponding EOM is given in Table 3. In case 2, a more detailed count of tracer labeling was done. Specifically, tracer labeling was combined with anti-ChAT immunostaining, and the number of double-positive palisade endings versus the number of ChATpositive but tracer-negative palisade endings was counted. The percentage of BDA-labeled palisades varied between 7 and $46 \%$ with a mean of $26.5 \%$.

In neither the oculomotor cases nor the abducens cases did analyses of the trigeminal ganglion reveal tracer-positive cell bodies or axons. However, in the abducens cases tracer-positive axons were observed in the abducens nerve, which passes close to the trigeminal ganglion (Fig. 6). The ophthalmic, maxillary and mandibular branches of the trigeminal nerve contained no tracer. Likewise, the mesencephalic trigeminal nucleus showed no tracer labeling in the cell bodies (data not shown).

\section{The ophthalmic division of the} trigeminal ganglion and the ophthalmic branch of the trigeminal nerve do not contain cholinergic axons

To test whether cholinergic trigeminal afferents supply palisade endings, immunohistochemical characterizations of the trigeminal ganglion and the ophthalmic branch of the trigeminal nerve were performed. Specifically, sections of the trigeminal ganglion and the intracranial part of trigeminal nerve were stained with antiChAT and anti-neurofilament antibodies. Immunolabeling also included the abducens nerve, which passes close to the trigeminal ganglion.

Within the ophthalmic division of the trigeminal ganglion, all the perikarya were neurofilament-positive but ChATnegative (Fig. $7 A-C, F$ ). Furthermore, all the axons of the sensory ophthalmic nerve were neurofilament positive but lacked ChAT immunoreactivity (Fig. $7 A-D$ ). The sensory maxillary nerve and the sensory division of the mandibular nerve lacked ChAT immunoreactivity as well. Exclusively, the axons belonging to the motor fraction of the mandibular nerve (data not shown), and those of the abducens nerve exhibited ChAT and neurofilament immunoreactivity (Fig. 7C, E, F).

We also tested the CGRP immunoreactivity of the cat trigeminal ganglion. We found that the majority of trigeminal ganglion cells were CGRP immunonegative, although some perikarya appeared immunolabeled for this peptide (Fig. $7 G$ ). However, it is important to note that such cells lacked ChAT immunoreactivity (Fig. $7 B, C, F)$. Axons of the nearby abducens nerve exhibited CGRP immunoreactivity as well (Fig. $7 G$ ). In this case, the majority of motor axons (all of them ChAT immunoreactive) were also labeled for CGRP. Nevertheless, a few axons did not exhibit CGRP immunostaining (Fig. 7G, arrows). This finding is in agreement with previous data in the cat abducens nucleus showing that $\sim 80 \%$ of the ChAT-immunoreactive abducens motoneurons are also CGRP immunopositive (Moreno-Lopez et al., 1998; González-Forero et al., 2002).

\section{Discussion}

The present study represents the first combined analysis of the molecular characteristics, innervation pattern, and source of palisade endings in the cat. It indicates that palisade endings originate from EOM nuclei, presumably from MIF motoneurons, and are cholinergic and CGRP-positive. Figure 8 summarizes the motor-like properties of palisade endings according to our results. 
Table 3. Number of tracer-positive palisade endings in different extraocular muscles of cats 1,2,3, and 4 and counted number of palisade endings in cat 2

\begin{tabular}{|c|c|c|c|c|c|}
\hline \multirow[b]{2}{*}{ EOM } & \multicolumn{2}{|c|}{ Oculomotor injections } & \multicolumn{2}{|c|}{ Abducens injections } & \multirow[b]{2}{*}{ Cat $2^{b}$} \\
\hline & Cat $1^{a}$ & $\operatorname{Cat}^{a}$ & $\operatorname{Cat} 3^{a}$ & Cat $4^{a}$ & \\
\hline SR le/ric & $3 / 6$ & $5 / 2$ & $0 / 0$ & $0 / 0$ & $14 / 29$ \\
\hline IR le/ric & $5 / 13$ & $4 / 7$ & $0 / 0$ & $0 / 0$ & $20 / 19$ \\
\hline LR le/ric & $0 / 0$ & $0 / 0$ & $8 / 3$ & $6 / 4$ & \\
\hline MR le/ric & $7 / 10$ & $12 / 13$ & $0 / 0$ & $0 / 0$ & $40 / 57$ \\
\hline SO le/ric & $0 / 0$ & $0 / 0$ & $0 / 0$ & $0 / 0$ & \\
\hline 10 le/ric & $2 / 2$ & $7 / 1$ & $0 / 0$ & $0 / 0$ & $15 / 7$ \\
\hline Total & 48 & 51 & 11 & 10 & 191 \\
\hline
\end{tabular}

${ }^{a}$ Tracer-positive palisade endings.

${ }^{b}$ All counted.

'Abbreviations indicate the following muscles: SR, Superior rectus; IR, inferior rectus; LR, lateral rectus; MR, medial rectus; $\mathrm{SO}$, superior oblique; 10 , inferior oblique; LPS, levator palpebrae superioris. Left and right are indicated as le and ri, respectively.

Palisade endings have molecular characteristics of motor terminals

Recently, it has been demonstrated in several species (cat, monkey, sheep, and dog) that palisade endings exhibit ChAT immunoreactivity (Konakci et al., 2005a; Konakci et al., 2005b; Rungaldier et al., 2009a; Rungaldier et al., 2009b). Moreover, palisade endings in monkeys contain choline transporter and vesicular acetylcholine transporter and therefore show all the ingredients for the synthesis and storage of acetylcholine, the neurotransmitter usually found in motor terminals (Blumer et al., 2009). Here, we confirmed that palisade endings are cholinergic and have demonstrated for the first time that palisade endings are also CGRP immunoreactive like motor terminals on singly and multiply innervated muscle fibers in cat EOMs. CGRP has been detected in spinal/cranial motoneurons (Forsgren et al., 1993; Moreno-López et al., 1998; González-Forero et al., 2002) as well as in guinea pig sensory trigeminal ganglion cells (Aigner et al., 1997). We also observed some cells containing CGRP in the trigeminal ganglion of cats, but these elements were noncholinergic and thus cannot contribute to the formation of palisade endings. We therefore conclude that CGRP detection in palisade endings is more compatible with motor, as opposed to sensory, properties (Fig. 8, see ChAT and CGRP).

\section{Palisade endings originate from EOM motor nuclei}

The present study provided direct evidence that the source of palisade endings lies in the EOM motor nuclei (Fig. 8). Our quan- titative analysis revealed that complete tracer labeling was not obtained for all palisade endings, and the extent of tracer labeling varied among the different EOMs. Labeling variations between muscles likely resulted from differential tracer involvement of the oculomotor subnuclei. The lack of complete labeling in the nerves can be explained by incomplete tracer uptake, failure of the BDA to label the entire axon, or, alternatively, that the duration of tracer transport was not the same for all axons to all muscles. Nevertheless, on the qualitative level our results clearly showed that palisade endings were anterogradely labeled after tracer injection into the EOM motor nuclei. Since all palisade endings have the same morphology and molecular phenotype, it is unlikely that palisade endings without tracer would differ in their central origin.

That palisade endings have a motor origin in cats is in line with similar neuronal tracing experiments in monkeys (Lienbacher et al., 2011a; Zimmermann et al., 2011), and this expansion to a second species strongly suggests that palisade endings in mammals have a common source. Evidence that palisade endings have a motor origin also comes from an early degeneration experiment. Sas and Scháb (1952) made lesions of the oculomotor nucleus in cats and found that, in addition to the expected loss of motor endplates on the EOMs, the palisade endings degenerated. However, in a prior cat study where neuronal tracer was injected into the trigeminal ganglion or into the EOM motor nuclei, only the trigeminal ganglion injections were reported to label structures resembling palisade endings (Billig et al., 1997). Since our tracer experiments disagreed with that previous work, we applied an additional test. We reasoned that since palisade endings are cholinergic structures, the trigeminal ganglion should contain cholinergic cell bodies if trigeminal axons form palisade endings. The present work demonstrated that this is not the case (Fig. 7). Furthermore, the ophthalmic branch of the trigeminal nerve, which had been supposed to carry axons of EOM proprioceptors in various mammals, including cat, was noncholinergic and not labeled with BDA (Manni et al., 1966; Porter and Spencer, 1982; Porter et al., 1983). Perhaps in the previous cat study, EOM motor nerves were contaminated by tracer due to their nearby course (Theisen et al., 1996).

\section{Palisade endings arise from MIF axons}

A MIF motor fiber/palisade ending construction was observed in $25 \%$ of the cases in cats, which was similar to our previous mon-
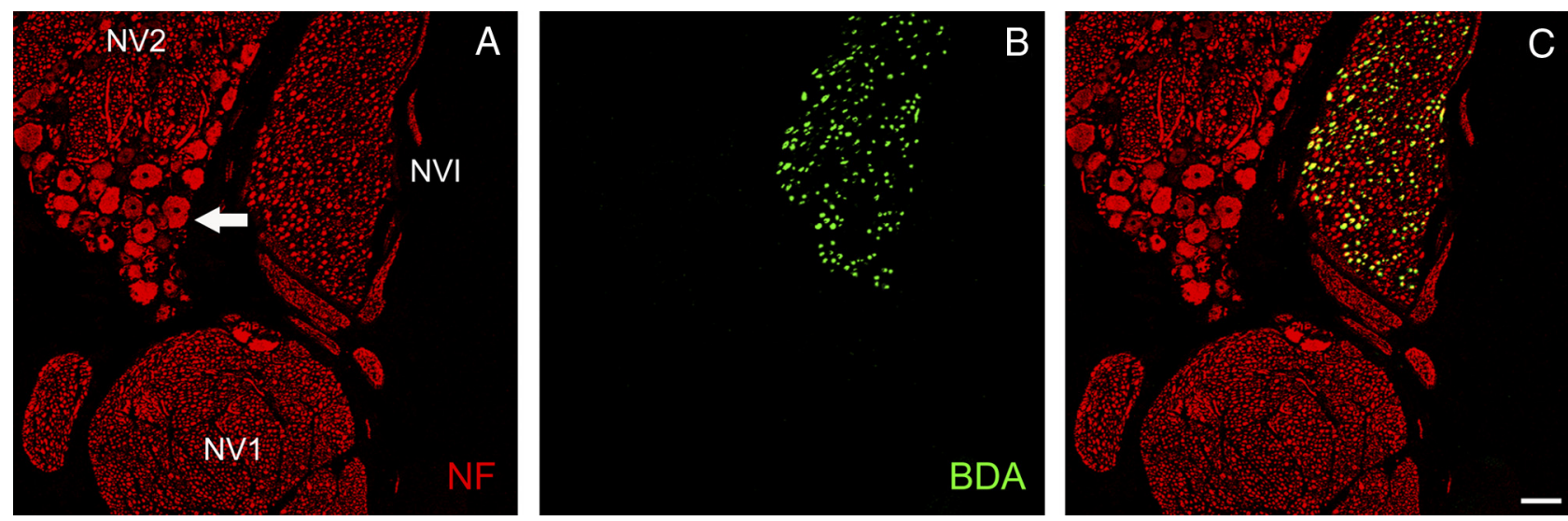

Figure 6. CLSM images close to the trigeminal ganglion following the injection of the anterograde BDA tracer into the abducens nucleus (cat 4). Images from a single optical section show the ophthalmic (NV1) and maxillary (NV2) nerve divisions, as well as the abducens nerve (NVI). A few cell bodies belonging to the maxillary division of the trigeminal ganglion are also visible (arrow) $\boldsymbol{A}$ - $\boldsymbol{C}$, Staining with anti-neurofilament (red) and BDA tracer visualization with streptavidin (green) $\boldsymbol{A}$, All the components are stained by the antibody to neurofilament protein. $\boldsymbol{B}, 0$ nly the abducens nerve is tracer-positive. $\boldsymbol{C}$, Overlay of $\boldsymbol{A}$ and $\boldsymbol{B}$ showing that neurofilament/tracer-positive axons in yellow mixed color are limited to the abducens nerve. Scale bar, $100 \mu \mathrm{m}$. 

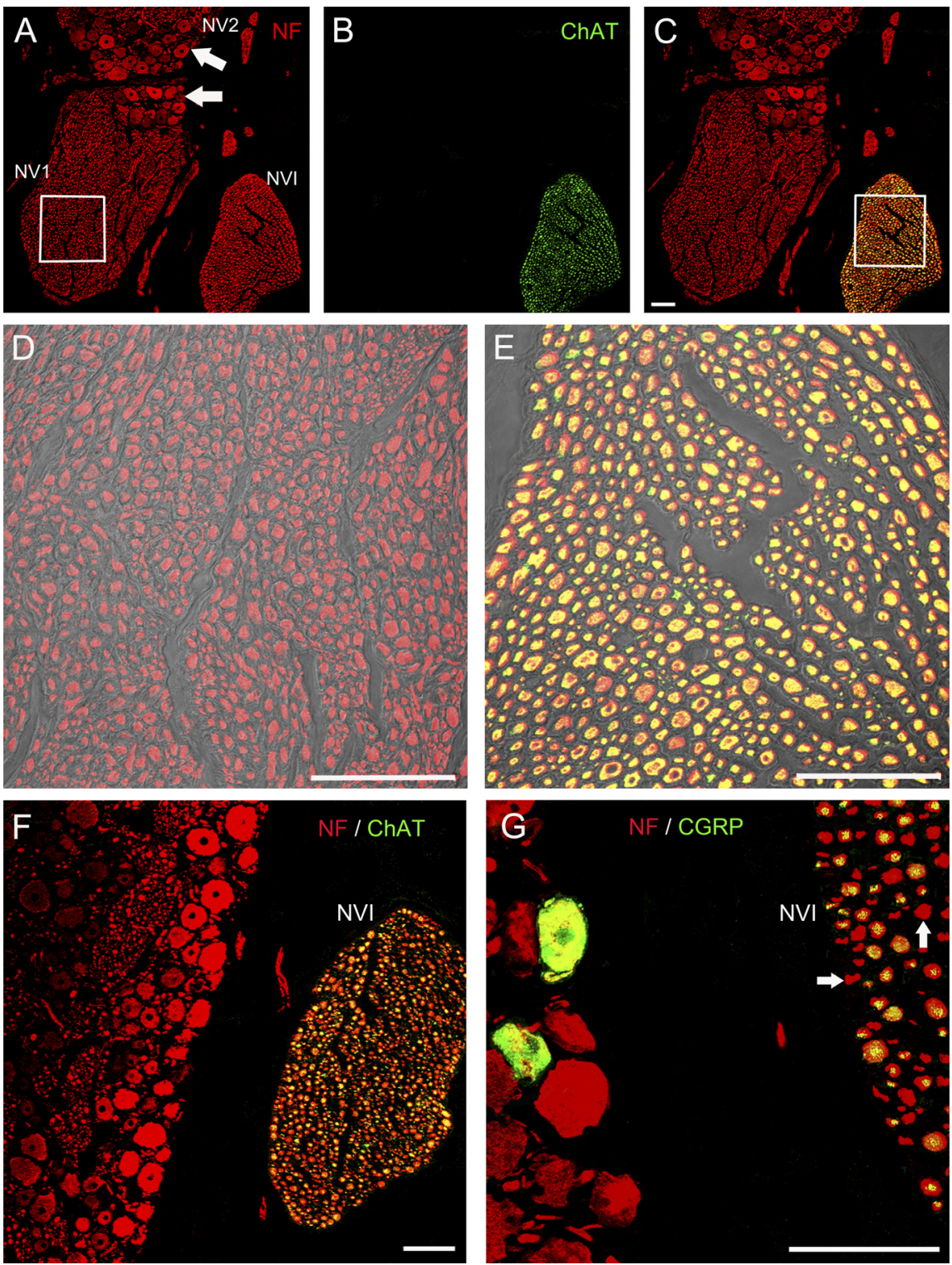

Figure 7. CLSM images from a single optical section showing branches of the trigeminal nerve [ophthalmic (NV1) and part of the maxillary (NV2) nerve divisions], with the abducens nerve (NVI) running nearby. A few cell bodies belonging to the ophthalmic and maxillary division of the trigeminal ganglion are also visible (arrows) $\boldsymbol{A}-\boldsymbol{F}$, Staining with anti-neurofilament (red) and antiChAT (green). G, Staining with anti-neurofilament and anti-CGRP. A, Axons in the ophthalmic and maxillary nerves and their respective perikarya exhibit neurofilament immunoreactivity, as do abducens nerve axons. $\boldsymbol{B}$, Note that only the abducens nerve is ChAT positive. $\boldsymbol{C}$, Overlay of $\boldsymbol{A}$ and $\boldsymbol{B}$ shows that only the abducens nerve contains doubly labeled axons in yellow mixed color of red neurofilament and green ChAT. $\boldsymbol{D}, \boldsymbol{E}$, Details (white squares) captured with high magnification of the ophthalmic nerve $(\boldsymbol{D})$ and abducens nerve $(\boldsymbol{E})$. The CLSM images were overlaid with a light-transmitted image. $\boldsymbol{F}$, Showing the ChAT-positive abducens nerve (NVI) and ChAT-negative perikarya of the ophthalmic division of the trigeminal ganglion. $\mathbf{G}$, In an adjacent section two CGRPpositive perikarya in yellow mixed color of red neurofilament and green (GRP are visible. The axons of the nearby abducens nerve (NVI) are (GRP immunoreactive as well (yellow mixed color). A few axons (arrow), however, lacked CGRP. Scale bars, $100 \mu \mathrm{m}$.

key data in which such a feature was found in $30 \%$ of the cases (Blumer et al., 2009). Whether this innervation pattern is actually the general scheme for palisade endings is a critical unanswered question.

Despite their origin in the EOM motor nuclei and their connection to MIF axons, it has been suggested that palisade endings still might have a sensory function (Lienbacher et al., 2011b). In monkeys following comparison of central and distal EOM tracer injections, two morphologically distinct populations of retrogradely labeled neurons were detected in separate groups around the periphery of the EOM motor nuclei, one multipolar and the other consisting of round and spindleshaped cells. It was proposed that the latter group, which supplies the tendinous insertion, might be similar to mesencephalic trigeminal neurons, innervating palisade endings peripherally and giving off collaterals that terminate centrally (Lienbacher et al., 2011b). However, there is currently no evidence that these neurons display central axon collaterals or that the oculomotor nucleus projects to the trigeminal sensory nuclei. The latter would seem necessary if these cells were the source of central sensory signals for eye position. The present study does not directly address the question of two types of non-SIF efferent neurons, but the results do not support this dichotomy. Both the immunolabeling and tracer labeling revealed axons forming palisade endings that also established multiple nerve terminals along the muscle fibers. Such neuromuscular contacts were undoubtedly motor, as demonstrated by their $\alpha$-bungarotoxin binding, and resembled en grappe endings usually found on MIFs (Harker, 1972). The only possible interpretation of the present data is that at least some MIF motoneurons supply palisade endings, as outlined in Figure 8.

\section{Palisade endings share ultrastructural features with classical proprioceptors, but display different patterns of molecular characteristics}

Palisade endings are enigmatic structures since, despite their clear motor characteristics, they exhibit sensory features as well. Specifically, palisade nerve terminals contact the collagen bundles of the tendon, which is in analogy to Golgi tendon organs (Ruskell, 1978; Alvarado-Mallart and Pinçon-Raymond, 1979; Richmond et al., 1984; Blumer et al., 1998; Rungaldier et al., 2009a). In fact, very few palisade nerve terminals were observed contacting the muscle fiber in sheep and cats. When such neuromuscular contacts were present, they lacked a basal membrane. This is reminiscent of sensory nerve terminals in muscle spindles (Alvarado-Mallart and Pinçon-Raymond, 1979; Blumer et al., 1998). However, differences between palisade endings and classical proprioceptors with respect to molecular properties are evident. Specifically, in sheep EOMs sensory nerve terminals in muscle spindles and Golgi tendon organs are noncholinergic in contrast to the cholinergic phenotype of palisade endings (Rungaldier et al., 2009b).

The function of palisade endings is unexplained at present Based on molecular characteristics, connectional patterns, and innervation morphology, the present results argue that palisade endings should not be interpreted as sensory organs (Fig. 8). 
However, a functional rationale for the action of palisade endings is difficult to find. Upon excitation, neurotendinous contacts in palisade endings presumably release acetylcholine, but it remains unclear what effect, if any, neurotransmitter release would have on collagen fibrils. Maybe receptors for acetylcholine are present at the muscle fiber tip, either at very low densities (undetectable by the $\alpha$-bungarotoxin staining) or as yet unidentified receptor proteins. Thus, when a MIF motoneuron forming palisade endings fires, palisade endings would allow muscle fiber contraction just at the terminal part of the muscle fiber, while en grappe endings would elicit contraction of the muscle fiber body. Contraction just at the fiber end would create only small muscle forces, but this may be necessary for the fine alignment of gaze or to maintain extreme eye positions.

An eye position signal has been identified in the primary somatosensory cortex of monkey (Wang et al., 2007), and loss of sensory input to the orbit can affect oculomotor and other behaviors (Fiorentini et al., 1986; Knox et al., 2000) even though classical proprioceptors are nearly absent. Moreover, when palisade endings are damaged following strabismus surgery, patients exhibit deficits in spatial perception (Steinbach and Smith, 1981; Steinbach et al., 1987). These data suggest that eye muscles must have some type of sensory receptors. Possibly information from nonspecialized receptors, like those reported by Billig et al. (1997), enters the brain through conventional trigeminal pathways (Porter and Donaldson, 1991) and is combined with information on the pulling direction of the innervated muscles to produce the eye position signals recorded in the somatosensory cortex and elsewhere (Donaldson, 2000; Wang et al., 2007;). The need for a muscle spindle in an EOM that always experiences the same load is unclear, so a less specialized sensory system may be sufficient.

The present study complements our prior immunohistochemical findings and does not support the hypothesis that palisade endings are sensory proprioceptive organs. Clearly, physiological and cell biological investigation of palisade endings is imperative to identify their function.

\section{References}

Aigner M, Lukas JR, Denk M, Mayr R (1997) Sensory innervation of the guinea pig extraocular muscles: a 1,1'-dioctadecyl-3,3,3'3'-tetramethylindocarbocyanine perchlorate tracing and calcitonin gene-related peptide immunohistochemical study. J Comp Neurol 380:16-22. CrossRef Medline

Alvarado-Mallart RM, Pinçon-Raymond M (1979) The palisade endings of cat extraocular muscles: a light and electron microscope study. Tissue Cell 11:567-584. CrossRef Medline

Billig I, Buisseret Delmas C, Buisseret P (1997) Identification of nerve endings in cat extraocular muscles. Anat Rec 248:566-575. CrossRef Medline

Blumer R, Lukas JR, Wasicky R, Mayr R (1998) Presence and structure of innervated myotendinous cylinders in sheep extraocular muscle. Neurosci Lett 248:49-52. CrossRef Medline

Blumer R, Konakci KZ, Pomikal C, Wieczorek G, Lukas JR, Streicher J (2009) Palisade endings: cholinergic sensory organs or effector organs? Invest Ophthalmol Vis Sci 50:1176-1186. CrossRef Medline

Büttner-Ennever JA, Eberhorn A, Horn AK (2003) Motor and sensory innervation of extraocular eye muscles. Ann N Y Acad Sci 1004:40-49. CrossRef Medline
Dogiel A (1906) Die Endigungen der sensiblen Nerven in den Augenmuskeln und deren Sehnen beim Menschen und den Saeugetieren. Arch Mikroskop Anal 68:501-526. CrossRef

Donaldson IM (2000) The functions of the proprioceptors of the eye muscles. Philos Trans R Soc Lond B Biol Sci 355:1685-1754. CrossRef Medline

Fiorentini A, Cenni MC, Maffei L (1986) Impairment of stereoacuity in cats with oculomotor proprioceptive deafferentation. Exp Brain Res 63:364-368. Medline

Forsgren S, Bergh A, Carlsson E, Thornell LE (1993) Calcitonin generelated peptide expression at endplates of different fibre types in muscles in rat hind limbs. Cell Tissue Res 274:439-446. CrossRef Medline

González-Forero D, De La Cruz RR, Delgado-García JM, Alvarez FJ, Pastor AM (2002) Correlation between CGRP immunoreactivity and firing activity in cat abducens motoneurons. J Comp Neurol 451:201-212. CrossRef Medline

Harker DW (1972) The structure and innervation of sheep superior rectus and levator palpebrae extraocular muscles. I. Extrafusal muscle fibers. Invest Ophthalmol Vis Sci 11:956-969. Medline

Knox PC, Weir CR, Murphy PJ (2000) Modification of visually guided saccades by a nonvisual afferent feedback signal. Invest Ophthalmol Vis Sci. 41:2561-2565. Medline

Konakci KZ, Streicher J, Hoetzenecker W, Blumer MJ, Lukas JR, Blumer R (2005a) Molecular characteristics suggest an effector function of palisade endings in extraocular muscles. Invest Ophthalmol Vis Sci 46: 155-165. CrossRef Medline

Konakci KZ, Streicher J, Hoetzenecker W, Haberl I, Blumer MJ, Wieczorek G, Meingassner JG, Paal SL, Holzinger D, Lukas JR, Blumer R (2005b) Palisade endings in extraocular muscles of the monkey are immunoreactive for choline acetyltransferase and vesicular acetylcholine transporter. Invest Ophthalmol Vis Sci 46:4548-4554. CrossRef Medline

Lienbacher K, Mustari M, Ying HS, Büttner-Ennever JA, Horn AK (2011a) Do palisade endings in extraocular muscles arise from neurons in the motor nuclei? Invest Ophthalmol Vis Sci 52:2510-2519. CrossRef Medline

Lienbacher K, Mustari M, Hess B, Büttner-Ennever J, Horn AK (2011b) Is there any sense in the Palisade endings of eye muscles? Ann N Y Acad Sci 1233:1-7. CrossRef

Lukas JR, Blumer R, Denk M, Baumgartner I, Neuhuber W, Mayr R (2000) Innervated myotendinous cylinders in human extraocular muscles. Invest Ophthalmol Vis Sci 41:2422-2431. Medline

Maier A, DeSantis M, Eldred E (1974) The occurrence of muscle spindles in extraocular muscles of various vertebrates. J Morphol 143:397-408. CrossRef Medline

Manni E, Bortolami R, Desole C (1966) Eye muscle proprioception and the semilunar ganglion. Exp Neurol 16:226-236. CrossRef Medline

Moreno-López B, de la Cruz RR, Pastor AM, Delgado-García JM, Alvarez FJ (1998) Effects of botulinum neurotoxin type A on the expression of 
gephyrin in cat abducens motoneurons. J Comp Neurol 400:1-17. CrossRef Medline

Porter JD, Donaldson IM (1991) The anatomical substrate for cat extraocular muscle proprioception. Neuroscience 43:473-481. CrossRef Medline

Porter JD, Spencer RF (1982) Localization of morphology of cat extraocular muscle afferent neurones identified by retrograde transport of horseradish peroxidase. J Comp Neurol 204:56-64. CrossRef Medline

Porter JD, Guthrie BL, Sparks DL (1983) Innervation of monkey extraocular muscles: localization of sensory and motor neurons by retrograde transport of horseradish peroxidase. J Comp Neurol 218:208219. CrossRef Medline

Richmond FJ, Johnston WS, Baker RS, Steinbach MJ (1984) Palisade endings in human extraocular muscles. Invest Ophthalmol Vis Sci 25:471-476. Medline

Rungaldier S, Pomikal C, Streicher J, Blumer R (2009a) Palisade endings are present in canine extraocular muscles and have a cholinergic phenotype. Neurosci Lett 465:199-203. CrossRef Medline

Rungaldier S, Heiligenbrunner S, Mayer R, Hanefl-Krivanek C, Lipowec M, Streicher J, Blumer R (2009b) Ultrastructural and molecular biologic comparison of classic proprioceptors and palisade endings in sheep extraocular muscles. Invest Ophthalmol Vis Sci 50:56975706. CrossRef Medline
Ruskell GL (1978) The fine structure of innervated myotendinous cylinders in extraocular muscles of rhesus monkeys. J Neurocytol 7:693-708. CrossRef Medline

Sas J, Scháb R (1952) Die sogenannten "Palisaden-Endigungen" der Augenmuskeln. Acta Morph Acad Sci (Hungary) 2:259-266.

Steinbach MJ, Smith DR (1981) Spatial localization after strabismus surgery: evidence for inflow. Science 213:1407-1409. CrossRef Medline

Steinbach MJ, Kirshner EL, Arstikaitis MJ (1987) Recession vs marginal myotomy surgery for strabismus: effects on spatial localization. Invest Ophthalmol Vis Sci 28:1870-1872. Medline

Theisen SK, Podell M, Schneider T, Wilkie DA, Fenner WR (1996) A retrospective study of cavernous sinus syndrome in 4 dogs and 8 cats. J Vet Intern Med 10:65-71. CrossRef Medline

Wang X, Zhang M, Cohen IS, Goldberg ME (2007) The proprioceptive representation of eye position in monkey primary somatosensory cortex. [see comment]. Nat Neurosci 10:640-646. CrossRef Medline

Zelená J, Soukup T (1977) The development of Golgi tendon organs. J Neurocytol 6:171-194. CrossRef Medline

Zimmermann L, May PJ, Pastor AM, Streicher J, Blumer R (2011) Evidence that the extraocular motor nuclei innervate monkey palisade endings. Neurosci Lett 489:89-93. CrossRef Medline 